\title{
Vasoactive intestinal peptide ameliorates renal injury in a pristane-induced lupus mouse model by modulating Th17/Treg balance
}

\author{
Dongdong $\mathrm{Fu}^{\dagger}$, Soulixay Senouthai ${ }^{\dagger}$, Junjie $\mathrm{Wang}^{\dagger}$ and Yanwu You ${ }^{*}$ (D)
}

\begin{abstract}
Background: Lupus nephritis (LN) is an inflammation of the kidneys and is a major cause of mortality in systemic lupus erythaematosus (SLE) patients. In addition, Th17/Treg balance is one of the most important factors that can promote the development of $L N$. It has been reported that vasoactive intestinal peptide (VIP) is associated with the downregulation of both inflammatory and autoimmune diseases through regulating $T$ lymphocyte balance. Therefore, the aim of this study was to determine the role of VIP in modulating Th17/Treg balance in LN.

Methods: LN was induced in BALB/C female mice by injection pristane. After 3 months, mice were randomly divided into four groups: control, VIP + control, LN and VIP + LN. Autoantibody levels were tested by ELISA. The distribution of Th17/Treg cells in vivo and in vitro was detected by FC. Renal tissues were examined by PASM and DIF for pathology and Foxp $3^{+} \mathrm{CD}^{+}$. The mRNA and protein expression levels of pro- and anti-inflammatory cytokines were detected by qRT-PCR and western blotting.

Results: VIP can improve renal injury by regulating Th17/Treg imbalance in LN mice. Proteinuria, renal function defects and autoantibodies were significantly decreased, and Th17/Treg cell balance was restored in VIP compared with LN mice. In addition, VIP improved renal lesions by promoting the expression of Foxp $3^{+} \mathrm{CD}^{+}$in renal tissue. Furthermore, VIP downregulated the mRNA and protein expression of IL-17, IL-6 and upregulated Foxp3, IL-10 expression.

Conclusions: VIP reduced LN proteinuria and renal function defects and restored the Th17/Treg cell balance. Furthermore, VIP also downregulated autoantibody and inflammatory cytokine expression and upregulated Foxp3 and IL-10 expression.
\end{abstract}

Keywords: Vasoactive intestinal peptide, Th17/Treg balance, Lupus nephritis

\section{Background}

Lupus nephritis (LN) is a disorder in which the body's immune system attacks the body's own cells and organs and is a major cause of mortality in systemic lupus erythaematosus (SLE) patients. Approximately 60\% of patients with proliferative SLE progress to end-stage renal disease (ESRD), which affects patient mortality, the

\footnotetext{
* Correspondence: youyanwu@163.com

${ }^{\dagger}$ Dongdong Fu, Soulixay Senouthai and Junjie Wang are co-first authors. Department of Nephrology, Affiliated Hospital of Youjiang Medical University for Nationalities, No. 18 Zhongshan Road II, Baise 533000, Guangxi Zhuang Autonomous Region, China
}

(c) The Author(s). 2019 Open Access This article is distributed under the terms of the Creative Commons Attribution 4.0 International License (http://creativecommons.org/licenses/by/4.0/), which permits unrestricted use, distribution, and reproduction in any medium, provided you give appropriate credit to the original author(s) and the source, provide a link to the Creative Commons license, and indicate if changes were made. The Creative Commons Public Domain Dedication waiver (http://creativecommons.org/publicdomain/zero/1.0/) applies to the data made available in this article, unless otherwise stated.

kidneys are functioning below 10\% of their normal function [1]. The cause of LN is not understood, and multifactorial interactions among various genetic and environmental factors are likely involved. Defective immune regulatory mechanisms, such as the impairment of $\mathrm{T}$ cell (Th17/Treg imbalance) function, are important contributors to the development of $\mathrm{LN}$ due to proinflammatory cytokine secretion, which greatly stimulates $\mathrm{B}$ cells for autoantibody production and contributes to organ damage.

Recently, accumulating evidence has suggested that Th17/Treg cell functional imbalance is closely associated 
with the pathogenesis of LN [2-4]. Th17 cells play a role in adaptive immunity to protect the body against pathogens, but they have also been implicated in the pathogenesis of tissue inflammation and tissue destruction, which play a critical role in many autoimmune and inflammatory disorders. In contrast, Treg cells can modulate effector $\mathrm{T}$ cell function to maintain immunological homeostasis and prevent autoimmunity. Thus, the disruption of Th17/Treg balance is considered the major pathogenic contributor to renal damage in LN. In addition, recovery of the immune balance between Th17 and Treg cells might help to ameliorate disease activity in LN.

Pristane, also known as hydrocarbon oil TMPD $(2,6$, 10, 14-tetramethylpentadecane), is capable of inducing a lupus-prone murine model that is similar to human SLE which includes female predominance, nephritis, arthritis and pulmonary vasculitis. In this model, autoantibody production and end-organ damage, including renal disease, depend on the IFN-I receptor signalling pathway. This model is widely used in research to understand the pathogenesis of LN and rheumatoid arthritis [5]. It has been reported that pristane-induced LN mice exhibit immunoregulatory abnormalities in their $\mathrm{T}$ cells and $\mathrm{B}$ cell hyperreactivity in in vitro immune responses [6].

Vasoactive intestinal peptide (VIP) is a 28 -amino acid peptide that is extensively distributed in tissues, which exerts pleiotropic functions in multiple systems, such as the gastrointestinal [7], cardiovascular [8], nervous [9], and immune $[10,11]$ systems. Many studies have shown that VIP can be used as an immunomodulatory and anti-inflammatory factor that participates in regulating immune balance $[10,12]$. It has been reported that VIP can effectively increase the number of Treg subpopulations while decreasing the number of Th17 subpopulations to improve inflammation in asthmatic mice by regulating Th17/Treg balance and VIP can also maintain the immune balance between Th17/Treg cells in the prevention and treatment of experimental autoimmune encephalomyelitis (EAE). Furthermore, VIP can stimulate the proliferation of Treg cells in vitro [13]. However, it remains unclear whether VIP regulates the immune balance of Th17/Treg cells in the pathogenesis of LN.

Therefore, in the present study, we investigated whether VIP might play an important role in the treatment of autoimmune diseases, including LN. To demonstrate the underlying mechanisms of VIP treatment in LN mice, we examined the dynamics of Th17/Treg cells in pristane-induced LN mice and investigated the therapeutic effects of VIP in this model. The present study showed that VIP treatment could effectively ameliorate $\mathrm{LN}$, which might be a consequence of restoring the Th17/Treg balance in pristane-induced LN mice. Collectively, the findings from this study provide new clinical applications for VIP in the treatment of $\mathrm{LN}$.

\section{Methods}

\section{Reagents}

VIP (bs-0077P, Bioss Inc., Beijing) was dissolved in phosphate-buffered saline (PBS, Solabio, Beijing). Pristane $\left(\mathrm{C}_{19} \mathrm{H}_{40}\right)$ was purchased from Sigma-Aldrich (P2870, St. Louis, MO, USA). Fast Quant RT Super Mix and SYBR Green PCR reagents were purchased for quantitative RTPCR assays (R6424 and R6306, Tiangen, China). Antibodies for flow cytometry analysis were purchased from BD Biosciences (560,767, San Diego, USA). Enzyme-linked immunosorbent assay (ELISA) kits were purchased from Cusabio Biotech Co., Ltd., China. Flow cytometry detection of Treg and Th17 cell antibodies were from BD Biosciences (BD Biosciences, Cat\# 560767, San Diego, USA). CD3 were purchased from Abcam Biotech Co., Ltd., China (ab5690). Foxp3 were purchased from Santa Cruz Biosciences (sc-53, 876).

\section{In vivo studies \\ Mice}

Female specific pathogen-free BALB/c mice, aged 8 weeks and weighing $21 \pm 3 \mathrm{~g}$, were purchased from Model Animal Research Center of Nanjing University (Nanjing, China). All mice received sterilized food and water indefinitely in an SPF environment and were housed in light and dark within 12:12 h. A maximum of 5 mice per cage. All animal experiments SPF mice were Youjiang Medical Committee for approval, and every effort was made to minimize the damage to mice.

\section{Animal experimental design}

All female the mice that were reared were divided into four groups ( $n=5$ /group): (1) control group, BALB/c mice received a single injection of $0.5 \mathrm{~mL}$ normal saline intraperitoneally (i.p.); (2) VIP group, BALB/c mice were treated with $200 \mu \mathrm{g} /$ mouse VIP (once every 2 days, i.p.); (3) $\mathrm{LN}$ group, $\mathrm{BALB} / \mathrm{c}$ mice received a single injection of $0.5 \mathrm{~mL}$ pristane i.p. (Molecular formula: $\mathrm{C} 19 \mathrm{H} 40$, Molecular weight: 268.52, density: 0.783) [14]; and (4) LN + VIP group, BALB/c mice were injected with $0.5 \mathrm{ml}$ pristine, and after 3 months, $200 \mu \mathrm{g} /$ mouse VIP was injected intraperitoneally. After 4 weeks of treatment VIP, the female mice blood samples were collected by tail or retro-orbital puncture (using heparinized glass capillary tubes, EDTAK2) under anesthesia. The spleen and kidney were detached under sterile conditions. Spleen was collected in $1.5 \mathrm{~mL}$ tube with RPMI $1640(1 \mathrm{~mL})$. Renal pole fractions were snap frozen in liquid nitrogen and transversal slices of renal tissue were fixed in $10 \%$ formalin.

\section{Detection of proteinuria and renal function in mice}

Female mice were collected for $24 \mathrm{~h}$ in a metabolic cage, peripheral blood was collected from the posterior orbital venous plexus, and serum creatinine $(\mathrm{SCr})$ and blood 
urea nitrogen (BUN) values were measured after 4 weeks of completion of treatment as described previously [15].

\section{ELISA}

The serum of each group of mice was taken out from the ultra-low temperature freezer. The concentration of ANA, anti-dsDNA and anti-Sm antibodies in peripheral blood of each mouse was determined using an ELISA kit (Cusabio Biotech Co., Ltd.) in strict accordance with the instructions. Absorbance was measured with a TriStar LB 941 multimode microplate reader (Berthold Technologies, Bad Wildbad, Germany) at $450 \mathrm{~nm}$.

\section{Histopathological analysis}

To further assess renal pathological changes, right and left kidneys were collected from each of the sacrificed mice and further processed by immersion in 10\% neutral buffered formalin and conventional paraffin embedding, and stained with Periodic Acid-silver Methe-namine (PASM) according to standard procedures and examined under a light microscope. The average lesion areas were calculated with ImageJ software. Renal pathology examinations were performed in a blinded manner by a pathologist.

\section{Immunofluorescence staining}

For double staining with foxp3 and CD3 (for represent Tregs) [16], the sections were carried out using paraffinembedded sections $(4 \mu \mathrm{m})$ of renal tissues, and incubated with Foxp3 and CD3 primary antibodies individually overnight at $4{ }^{\circ} \mathrm{C}$. The next day, the samples were incubated with rabbit anti-mouse IgG secondary antibody for $1 \mathrm{~h}$ at room temperature. The samples were then observed with a fluorescence microscope (IX71, Olympus, Tokyo, Japan) equipped with ISCapture software, and images were taken with a CCD camera (Discovery C15, Olympus, Tokyo, Japan). The numbers of Foxp $3^{+}$positive (green) and $\mathrm{CD}^{+}$positive (red) cells counted under fluorescent microscopy.

\section{In vitro studies}

\section{Lymphocyte experimental design}

Mice spleen lymphocytes were separated in a sterile environment, adjusted to a cell concentration of $1 \times 10^{6} \%$ $\mathrm{mL}$ with RPMI-1640 medium containing $10 \%$ fetal bovine serum (Gibco), and cultured in 6-well plates. Subsequently, the stimulation factors IL- $6(50 \mathrm{ng} / \mathrm{mL})$, TGF- $\beta$ $(1 \mathrm{ng} / \mathrm{mL}), \mathrm{IL}-23(20 \mathrm{ng} / \mathrm{mL})$, anti-IFN $\gamma(3 \mu \mathrm{g} / \mathrm{mL})$ and anti-IL-4 $(3 \mu \mathrm{g} / \mathrm{mL})$ were added to induce Th17 cell proliferation, and TGF- $\beta(10 \mathrm{ng} / \mathrm{mL})$ and IL-2 $(10 \mathrm{ng} / \mathrm{mL})$ were added to stimulate Treg cell proliferation. After culturing in a $37^{\circ} \mathrm{C}, 5 \% \mathrm{CO}_{2}$ incubator for 3 days. The Treg cells were divided randomly into four groups: (1) NC group, Treg cells from BALB/c mice; (2) LN group, Treg cell from pristane-induced lupus mice; (3) VIP group, Treg cells from BALB/c mice were treated with $10^{-7} \mathrm{M}$ VIP; (4) LN + VIP group, Treg cell from pristane-induced lupus mice were treated with $10^{-7} \mathrm{M}$ VIP. The Th17 cells were divided randomly into four groups: (1) NC group, Th17 cells from BALB/c mice; (2) LN group, Th17 cell from pristane-induced lupus mice; (3) VIP group, Th17 cells from $\mathrm{BALB} / \mathrm{c}$ mice were treated with $10^{-7} \mathrm{M}$ VIP; (4) LN + VIP group, Th17 cell from pristane-induced lupus mice were treated with $10^{-7} \mathrm{M}$ VIP. The experiment was terminated after $72 \mathrm{~h}$ of treatment. The fractions of Treg and Th17 cells in each group were detected by flow cytometry. The mRNA and protein levels of IL-17, IL-6, Foxp3 and IL-10 were detected by qRT-PCR and western blotting.

\section{Flow cytometric analysis}

The spleen and peripheral blood are collected and the lymphocytes are separated and operated under aseptic conditions. The cell suspension was separated by density gradient centrifugation in mouse lymphocyte isolation medium (Solarbio, Cat\# P8860, Beijing, China). The cells of each group were washed three times with sterile PBS buffer. The lymphocyte suspensions $\left(1 \times 10^{6} / \mathrm{mL}\right)$ were stained with antibodies specific for mouse CD4, IL-17 (for Th17 cells) and Foxp3 (for Treg cells) or isotype-matched controls according to the manufacturer's instructions (Cat \#560767). The samples were mixed gently, incubated for $30 \mathrm{~min}$ at room temperature, and subsequently analysed using a flow cytometer (FC) FACS CantoII (BD Biosciences, San Jose, CA, USA).

\section{RNA purification and quantitative real-time PCR assays}

Renal tissue was collected in RNase-free centrifuge tube using Trizol reagent (lot \#103105, Invitrogen) to extract total RNA. For cDNA synthesis, $2 \mu \mathrm{g}$ purified total RNA was reverse-transcribed (KR116, Tiangen, Beijing, China). The expression levels of IL-17, IL-6, Foxp3, IL-10 and GAPDH were determined using SuperReal PreMix Plus (SYBR Green) (FP205, Tiangen, Beijing) according to the requirements of the manual, the data is used and tested in strict accordance with the specifications. The data was repeated three times for each experiment. The primer sequences are shown in Table 1. Comparative gene expression was calculated by the $2^{-\Delta \Delta \mathrm{Ct}}$ method as described previously [17].

\section{Protein isolation and western blot analysis}

The method for protein from total kidney homogenate was extracted according to the manufacturer's recommended protocol (Cwbiotech, Beijing, China). Proteins were resolved on $10 \%$ sodium dodecyl sulfate-polyacrylamide gel electrophoresis (SDS-PAGE) and transferred to nitrocellulose membranes (GE Healthcare, Freiburg, Germany). After transfer, 5\% skimmed milk powder was used to block the membranes at room temperature for $1 \mathrm{~h}$. Then, the 
Table 1 PCR primers used in this study

\begin{tabular}{lll}
\hline Gene Name & Forward(5'-3') & Reverse $\left(5^{\prime}\right.$ - $\left.^{\prime}{ }^{\prime}\right)$ \\
\hline IL-17 & TGATGCTGTTGCTGCTGCTGAG & TGGACGGTTGAGGTAGTCTGAGG \\
IL-6 & AGGAGTGGCTAAGGACCAAGACC & CTGACCACAGTGAGGAATGTCCAC \\
FOXP3 & GAAGAGCCTGCCTTGGTACATTCG & TGTGAAGGTCCAGTGCTGTTGC \\
IL-10 & CAAGGCAGTGGAGCAGGTGAAG & GCTCTGTCTAGGTCCTGGAGTCC \\
GAPDH & AACTTGGCATTGTGGAAGG & GGATGCAGGGATGATGTTCT \\
\hline
\end{tabular}

membranes were pre-incubated with primary Foxp3 (sc-53, 876, 1:500 dilution), IL-6 (sc-7920, 1:500 dilution) (Santa Cruz Biotechnology, California, USA), anti-IL-10 (ab9969, $1.5 \mu \mathrm{g} / \mathrm{mL}$ dilution), anti-IL-17 (ab79056, $1.5 \mu \mathrm{g} / \mathrm{mL}$ dilution) (Abcam Ltd., Hongkong, China) and GAPDH (1:1000 dilution) (Danvers, MA, USA) antibodies at $4{ }^{\circ} \mathrm{C}$ overnight and then incubated with secondary antibodies following three washes with TBST. Then, to detection of the expression level of the protein of interest using an enhanced chemiluminescence (ECL, Millipore, Billerica, MA, USA) western blot detection kit and a chemiluminescent imaging system (Bio-Rad, USA)..

\section{Statistical analysis}

All data values were mean \pm standard error of the mean in the bar graph. Measurements were performed by oneway ANOVA with Newman-Keuls multiple comparison post-test (for more than two comparisons) and paired t-test (comparison of two groups) in data comparison statistical significance analysis. All data were conducted with SPSS 17.0 software (SPSS Inc., Chicago, USA). P-values less than 0.05 were regarded significant.

\section{Results}

\section{An LN mouse model was successfully established}

Twelve weeks after pristane injection, significant increases in proteinuria $(\mathrm{mg} / 24 \mathrm{~h})(88.17 \pm 5.76)$ and serum $\mathrm{Cr}(1.39 \pm$ $0.05)$ and BUN $(25.71 \pm 4.12)$ levels were detected in $\mathrm{LN}$ mice compared to control mice $(25.33 \pm 5.48,0.25 \pm 0.05$ and $8.51 \pm 1.90$, respectively, $p<0.01)$. Furthermore, the ANA (63.69 \pm 3.56$)$, anti-dsDNA (78.52 \pm 4.04$)$ and anti-Sm $(51.89 \pm 2.17)$ autoantibodies were significantly higher in LN mice than in control mice $(29.42 \pm 3.25,28.58 \pm 1.99$ and $20.81 \pm 3.02$, respectively, $p<0.01$ ). According to these results, a mouse model of LN was established successfully, and these mice are used in the subsequent studies [18].

\section{VIP reduces autoantibody levels, proteinuria and renal function defects in LN mice}

After 4 weeks of VIP treatment, LN mice presented with significantly less renal injury, as demonstrated by marked reductions in urine protein $(\mathrm{mg} / 24 \mathrm{~h})$, renal function defects and autoantibodies. As shown in Fig. 1, the $24 \mathrm{~h}$ proteinuria (111.12 \pm 13.28$), \operatorname{SCr}(1.35 \pm 0.09)$ and BUN $(31.36 \pm 2.86)$ levels were higher in $\mathrm{LN}$ mice than in $\mathrm{LN}+\mathrm{VIP}$ mice
(71.12 $\pm 4.02,0.69 \pm 0.36$ and $19.36 \pm 1.86$, respectively, $p<$ 0.01 ) (Fig. 1a). The LN mice also exhibited significantly higher levels of ANA (67.89 \pm 5.10$)$, anti-dsDNA (81.52 \pm 6.39) and anti-Sm $(51.49 \pm 4.82)$ than the LN + VIP-treated mice $(51.49 \pm 4.82,53.68 \pm 3.90$ and $33.16 \pm 4.29$, respectively, $p<0.01$ ) (Fig. 1b). There were no significant differences between the control and VIP mice $(p>0.05)$.

\section{VIP modulated Th17/Treg imbalance in the peripheral blood and spleens of LN mice}

The number of Th17 cells was significantly increased in the peripheral blood and spleens of LN mice $(2.61 \pm 0.02$ and $6.82 \pm 0.13$, respectively) (Fig. 2a), while the number of Treg cells was significantly decreased in the peripheral blood and spleens $(0.53 \pm 0.08$ and $1.08 \pm 0.07$, respectively) (Fig. 2b) when compared with those in the control mice (Th17: $1.21 \pm 0.06$ and $2.45 \pm 0.23$ in the peripheral blood and spleen, respectively; Treg: $1.20 \pm 0.03$ and $3.09 \pm 0.07$ in the peripheral blood and spleen, respectively, $p<0.01)$. The results also suggest that after $\mathrm{LN}+$ VIP treatment, the number of Th17 cells was significantly decreased $(1.86 \pm 0.05$ in the peripheral blood and $4.05 \pm 0.07$ in the spleen), and the number of Treg cells was significantly increased $(0.98 \pm 0.07$ in the peripheral blood and $2.09 \pm 0.07$ in the spleen, $p<0.01$ ) when compared to those in the $\mathrm{LN}$ mice (Fig. 2). However, there were no significant differences between the control and VIP mice $(p>0.05)$. These findings demonstrated that VIP can inhibit Th17 cell differentiation and promote the generation of Treg cells, thereby restoring the Th17/ Treg cell balance and ameliorating LN in mice.

\section{VIP ameliorated the renal lesions in pristane-induced LN} mice

The PASM-stained renal tissues in LN mice appeared to have renal glomerular atrophy, basement membrane thickening and rupture, mesangial area widening, mesangial matrix increases, mesangial cell proliferation (more than $55 \%$ ), and renal tubule necrosis. In the LN + VIP-treated mice, histopathological changes in the renal cortical areas were significantly improved, including less mesangial matrix accumulation and decreased mesangial cell proliferation (less than 25\%) (Fig. 3). 


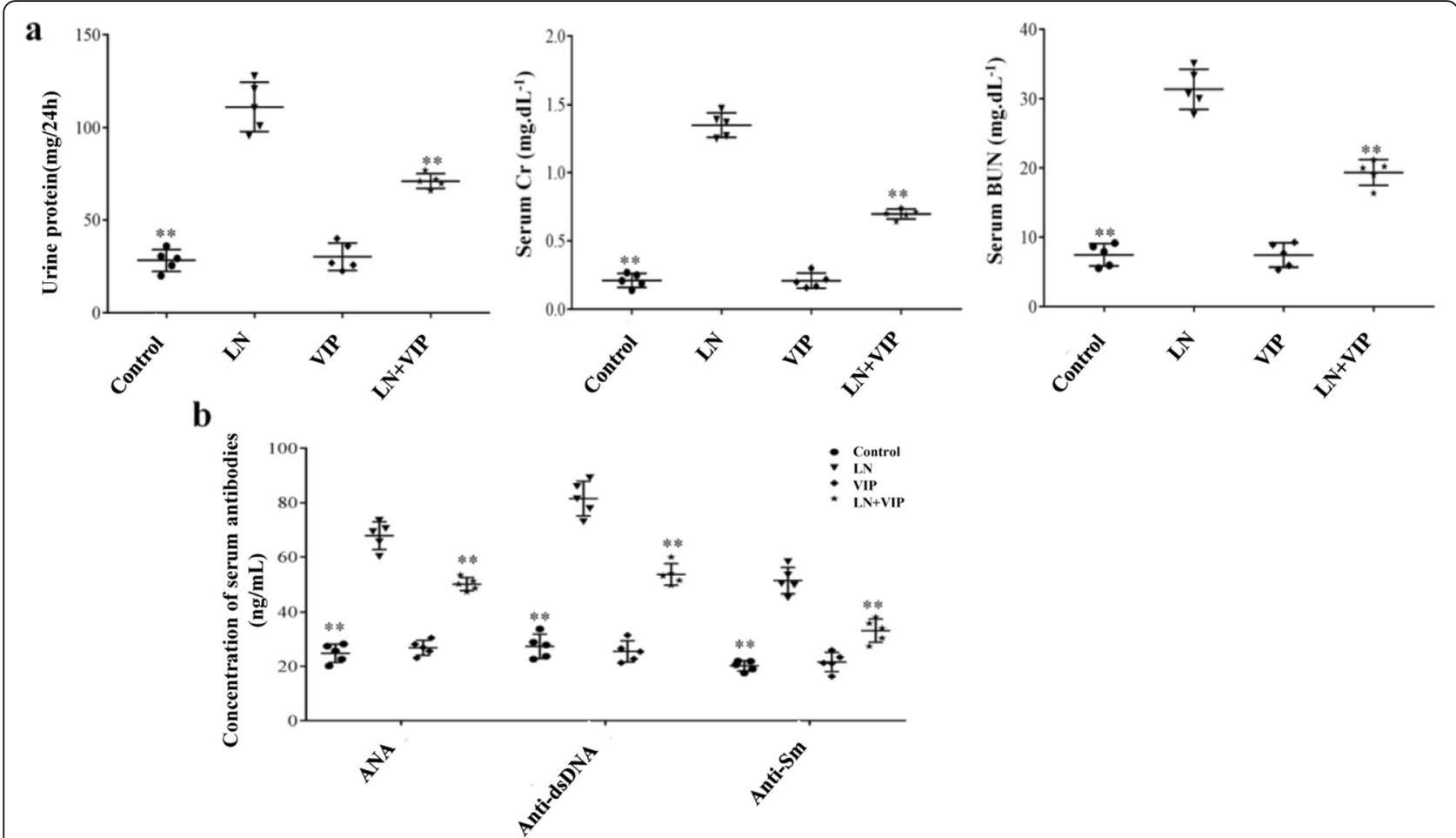

Fig. 1 VIP ameliorates the serum levels of autoantibody and renal function in LN mice. After 4 weeks of VIP treatment, urine was collected for $24 \mathrm{~h}$ using metabolic cages to determine the protein urine levels, and blood was collected for serum creatinine, BUN and autoantibody measurements. a Assessment of proteinuria and kidney function in mice and $\mathbf{b}$ serum levels of ANA, anti-dsDNA and anti-Sm antibodies. The data are expressed as the mean \pm standard deviation $(n=5)$. Statistical analyses were performed using one-way ANOVA. ${ }^{* *} p<0.01$ compared with LN mice

\section{Expression of Foxp3 and CD3 in renal tissue is regulated by VIP}

To determine the therapeutic effect of VIP on renal tissue in LN mice, we analysed kidney sections stained by DIF. VIP obviously improved the pristane-induced renal pathological changes in LN mice. The expression levels of Foxp3 and CD3 compared between the different groups of mice are shown in Fig. 4. According to the DIF staining of renal tissue with antibodies to Foxp3 and CD3 indicated that the expression of Foxp $3^{+} \mathrm{CD}^{+}$were lower in the interstitial cellular infiltrates of $\mathrm{LN}$ renal tissue than in the control mice, while the Foxp $3^{+} \mathrm{CD}^{+}$levels were increased after VIP treatment in LN + VIP mice compared to LN mice. These findings indicated that VIP could modulate Th17/Treg immune response and reduced renal injury in LN mice model.

VIP inhibited Th17 cell differentiation and promoted Treg cell proliferation in lymphocytes from the mouse spleens In this experiment, Th17 and Treg cells in lymphocytes from the spleen were determined by flow cytometric analysis. As shown in Fig. 5, the results show that the LN mice exhibited significantly higher levels of Th17 (5.15 \pm 0.18$)$ and lower levels of Treg cells $(0.97 \pm 0.02)$ than the control mice $(1.69 \pm 0.04$ and $2.09 \pm 0.06$, respectively, $p<0.01)$. The number of Th17 cells $(2.99 \pm 0.07)$ was significantly lower, and the number of Treg cells $(1.43 \pm 0.04)$ was significantly higher in LN + VIP mice than in LN mice $(p<0.01)$. However, there were no significant differences between the control and VIP mice $(p>0.05)$.

VIP regulated the gene and protein expression of pro- and anti-inflammatory cytokines in the renal tissue of LN mice IL-17, IL-6, Foxp3 and IL-10 mRNA and protein expression levels in renal tissues were analysed by qRT-PCR and western blotting. As shown in Fig. 6a, the mRNA and protein levels of IL-17 (3.39 \pm 0.40 and $0.98 \pm 0.09)$ and IL- $6(3.26 \pm 0.29$ and $0.96 \pm 0.04)$ were higher, and the mRNA and protein levels of Foxp3 $(0.25 \pm 0.07$ and $0.32 \pm 0.04)$ and IL-10 (0.42 \pm 0.03 and $0.19 \pm 0.03)$ were lower in the renal tissues of LN mice than in those of control mice (IL-17: $1.0 \pm 0.0$ and $0.26 \pm 0.03$; IL-6: $1.0 \pm 0.0$ and $0.59 \pm 0.05$; Foxp3: $1.0 \pm 0.0$ and $0.74 \pm 0.01$ and IL-10: $1.0 \pm 0.0$ and $0.54 \pm 0.01 ; p<0.05$ and $p<0.01)$. Compared to control treatment in LN mice, LN + VIP treatment significantly increased Foxp3 $(0.65 \pm 0.15$ and $0.82 \pm 0.03)$ and IL-10 (0.69 \pm 0.04 and $0.41 \pm 0.02)$ mRNA and protein expression and decreased IL-17 (1.97 $\pm 0.22,0.43 \pm 0.01)$ and IL- $6(1.84 \pm 0.06,0.26 \pm 0.01)$ mRNA and protein expression. There were no significant differences between the control and VIP mice $(p>0.05)$. 


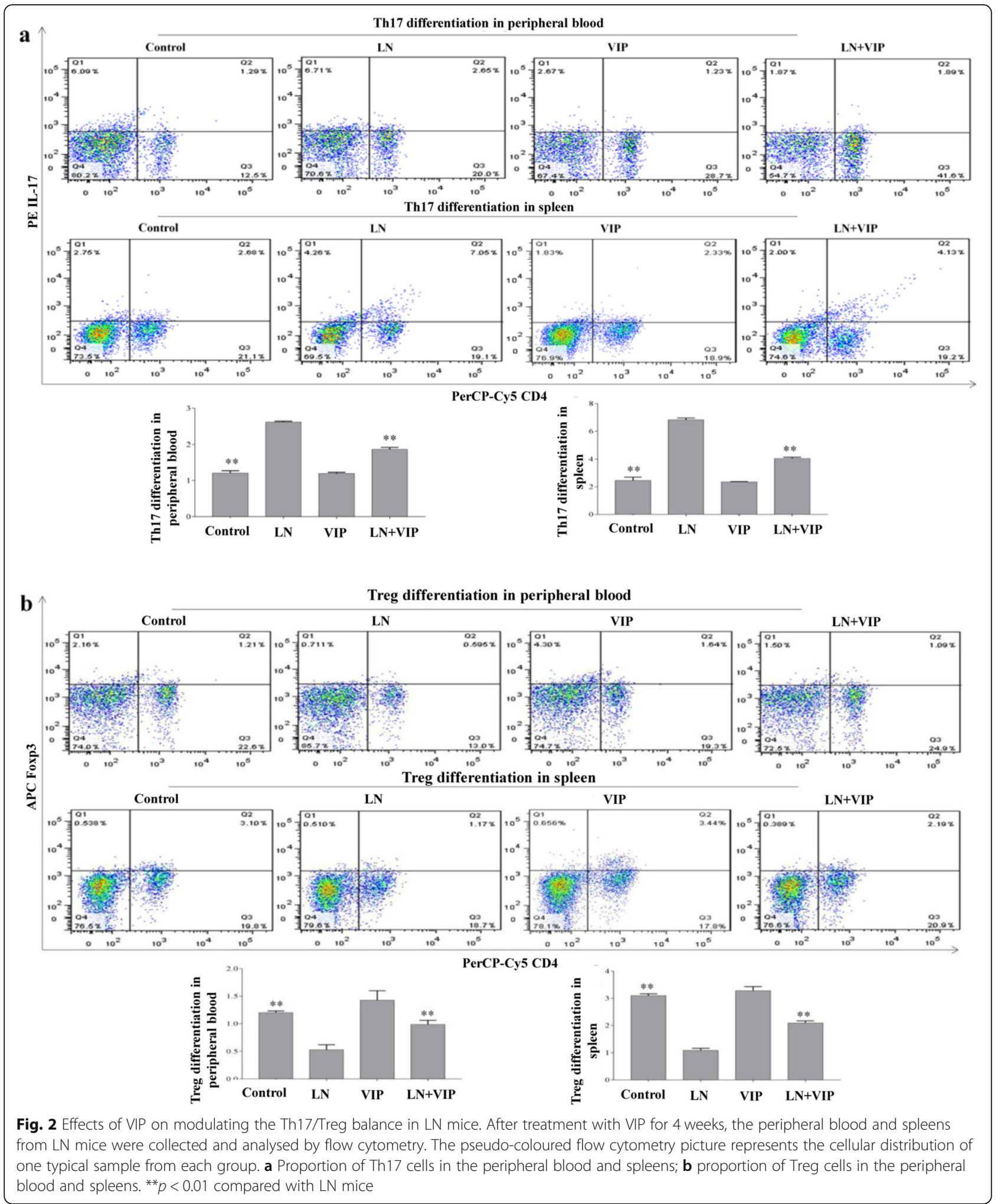




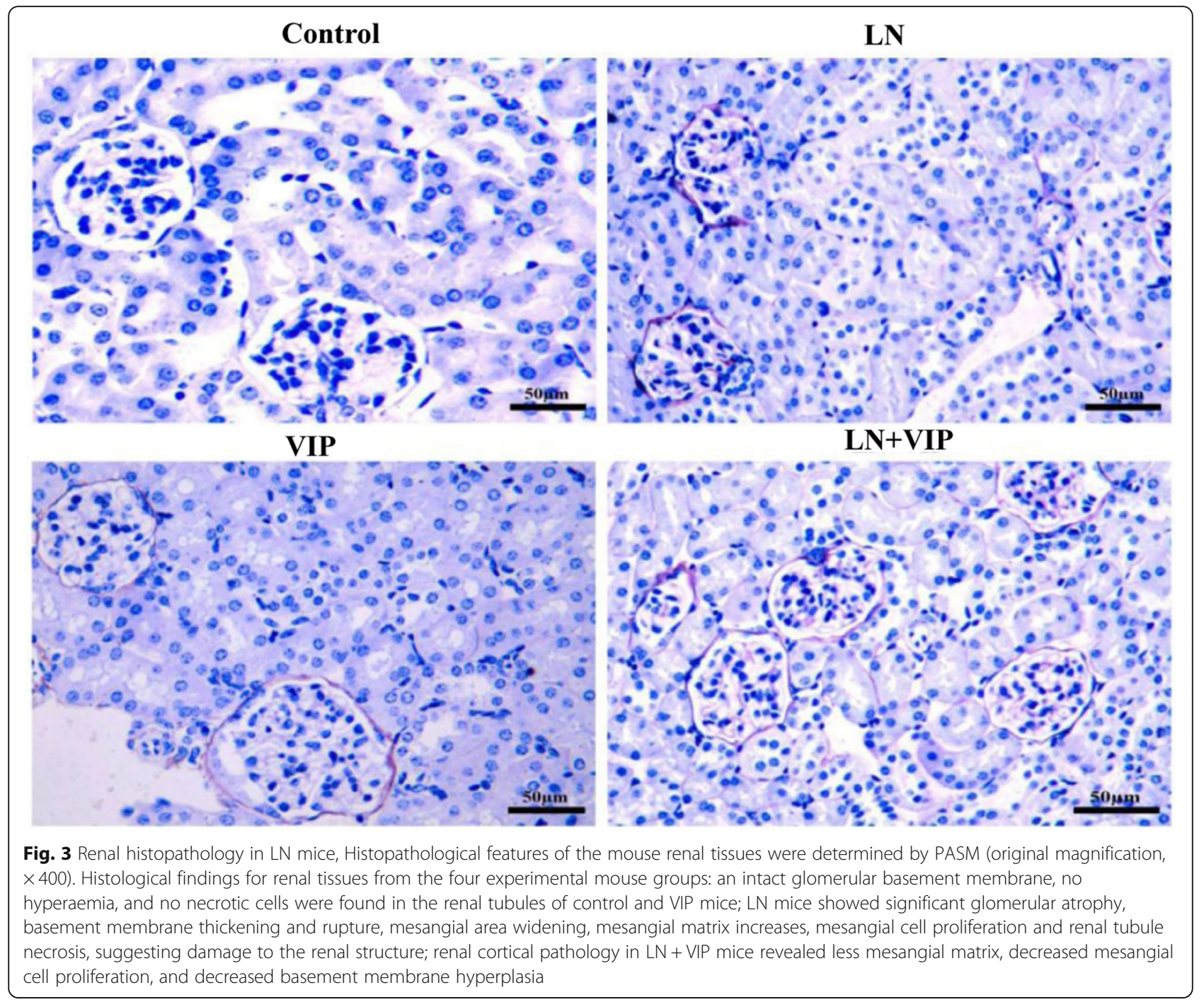

VIP regulated the gene and protein expression levels of pro- and anti-inflammatory cytokines in lymphocytes from $L N$ mice

To assess the effects of VIP on the lymphocyte response in LN mice, the mRNA and protein levels of IL-17, IL-6, Foxp3 and IL-10 in lymphocytes were examined by qRT-PCR and western blotting (Fig. 6b). These results were consistent with the mRNA and protein expression data from renal tissue. mRNA and protein expression levels of IL-17 (3.01 $\pm 0.30,1.15 \pm 0.14)$ and IL-6 (2.62 \pm $0.19,1.01 \pm 0.08$ ) were higher, and mRNA and protein expression levels of Foxp3 $(0.32 \pm 0.11,0.33 \pm 0.05)$ and IL-10 $(0.45 \pm 0.07,0.36 \pm 0.07)$ were lower in lymphocytes from LN mice than in those from control mice (IL-17: $1.0 \pm 0.0$ and $0.39 \pm 0.04 ;$ IL-6: $1.0 \pm 0.0$ and $0.49 \pm 0.04$; Foxp3: $1.0 \pm 0.0$ and $0.81 \pm 0.09$ and IL-10: $1.0 \pm 0.0$ and $0.82 \pm 0.08 ; p<0.05$ and $p<0.01$ ). Compared to control treatment in LN mice, LN + VIP treatment significantly increased Foxp3 $(0.66 \pm 0.10$ and $0.67 \pm$
$0.09)$ and IL-10 $(0.74 \pm 0.04$ and $0.90 \pm 0.99)$ mRNA and protein expression and decreased IL-17 (1.87 \pm 0.12 and $0.22 \pm 0.02)$ and IL-6 $(1.89 \pm 0.20$ and $0.48 \pm 0.05)$ mRNA and protein expression.

\section{Discussion}

The present study revealed that VIP can restore and maintain the immune balance between Th17 and Treg cells both in vivo and in vitro by inhibiting Th17 cell differentiation and restoring the number of Treg cells. These effects significantly reduce autoantibody production and inhibit the expression of proinflammatory cytokines while stimulating the expression of anti-inflammatory cytokines. These effects also correlated with reductions in renal function defects and proteinuria, as well as the amelioration of renal pathology. Importantly, our findings suggested that VIP is similarly effective for regulating the Th17/Treg balance in the treatment of LN mice. 

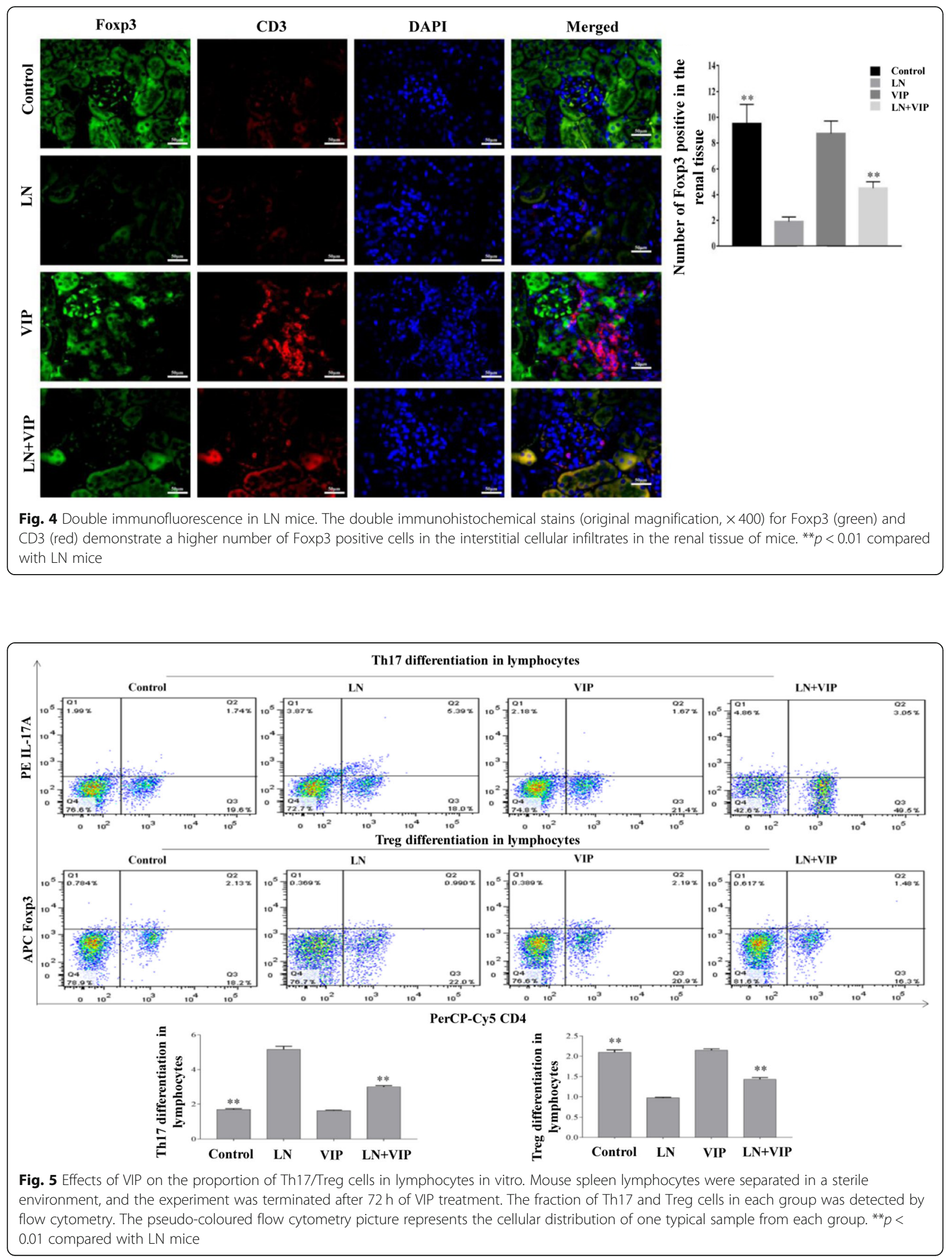


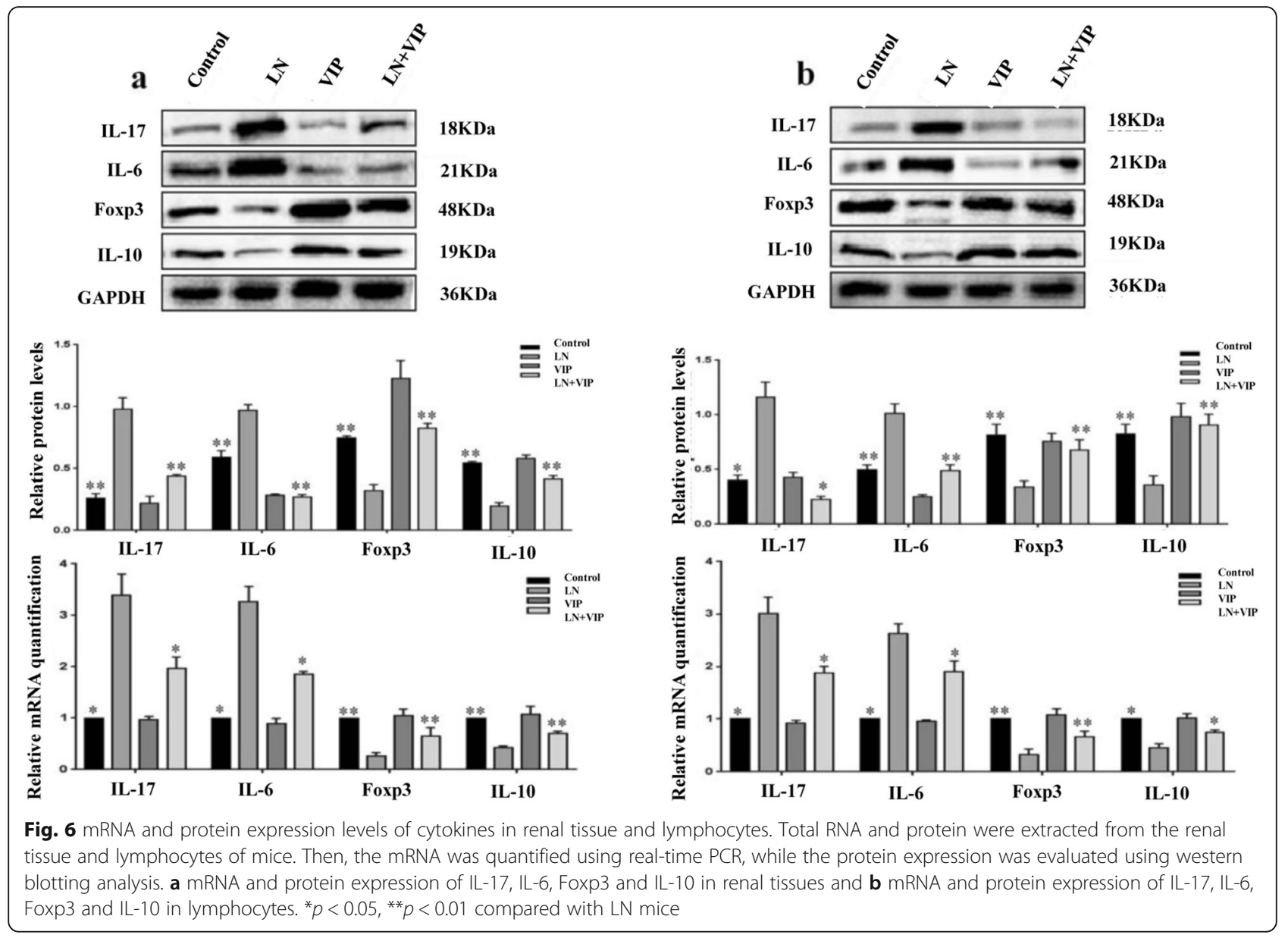

The distribution and function of $\mathrm{T}$ lymphocytes are some of the most important factors in the pathogenesis of autoimmune disorders. The pathogenesis of $\mathrm{LN}$ is a classical autoimmune disease characterized by $\mathrm{T}$ lymphocyte dysfunction, including Th17/Treg cell imbalance, which results in renal inflammation $[19,20]$. In recent years, immune balance disruptions of Th17/Treg cell subsets have gradually become a research hotspot. Many of these studies have found that Th17 cells induce autoimmunity, whereas Treg cells inhibit these phenomena and maintain immune homeostasis. The results from our study indicate that the peripheral blood and spleen have increased numbers of Th17 cells but decreased numbers of Treg cells. These cell changes result in enhanced IL-17 and IL-6 expression but reduced Foxp3 and IL-10 expression in the renal tissues of LN mice, which are significant risk factors for the occurrence of renal inflammation and underlying causes for developing renal damage. Therefore, the regulation of Th17/Treg cell balance might be used to restore immune homeostasis to provide therapeutic benefits in autoimmune conditions.

VIP is a neuropeptide that is widely distributed in the body and is a potent anti-inflammatory factor that affects the regulation strategies of both the innate and adaptive immune systems [21, 22]. Therefore, it has emerged as a potential candidate for the treatment of various autoimmune and inflammatory diseases; VIP has also been shown to be effective in the prevention of autoimmune diseases, such as diabetes mellitus, rheumatoid arthritis, EAE, sepsis and inflammatory bowel disease [11, 23-27]. Gonzalez-Rey et al. [28] indicated that VIP participates in the maintenance of immune tolerance to ameliorate autoimmune disease progression by expanding the population of Treg cells. Subsequently, Deng et al. [29] found that VIP inhibits inflammatory responses in autoimmune diseases by upregulating Treg cells, but it has also been associated with downregulating Th17 cells. Thus, the therapeutic effect of VIP has been attributed to its capacity to downregulate inflammatory factors by switching the Th17/Treg balance. Here, we provide additional evidence that VIP administration in vivo and in vitro can inhibit Th17 cells and stimulate the Treg response, which ameliorates renal function defects in LN mice, while simultaneously improving the inflammatory state, autoantibody expression and renal damage. 
This study enriched the understanding of the pathogenesis of LN and provided a theoretical basis for the application of VIP in LN. However, there are some limitations in present study. Any signalling pathways and transcription factors such as STAT3 and RORyt did not be explored. In order to explore the exact mechanism of VIP in LN, we are considering to detect some typical pathways or analyse the gene array in VIP-treated Th17 or Treg cells from lupus model to find out the differential signaling pathways and transcription factors, and even the interaction between them in the future study.

In summary, these experiments revealed that aberrant $\mathrm{T}$-cell homeostasis is a crucial event in LN pathogenesis, and Th17/Treg imbalance appears to be an important key pathogenic player. This imbalance leads to increased serum levels of autoantibodies and renal levels of IL-6 and IL-17, as well as renal function defects. Furthermore, decreased renal levels of Foxp3 and IL-10 are associated with glomerulonephritis in pristane-induced lupus mice. VIP is reliable and effective for treating $\mathrm{LN}$, and its therapeutic mechanisms should be effective for modulating Th17/Treg immune homeostasis by downregulating serum levels of autoantibodies and renal levels of IL-17 and IL-6 and upregulating renal levels of Foxp3 and IL-10, thus ameliorating renal function defects, proteinuria and renal damage.

\section{Conclusions}

VIP is a potential therapeutic agent for treating LN and other abnormal Th17/Treg diseases in mice. Therefore, promising results concerning the therapeutic targeting of Th17/Treg cell balance may open new lines of investigation for LN treatment in the near future. However, additional research is needed to further explain this process.

\section{Abbreviations}

EAE: Experimental autoimmune encephalomyelitis; ESRD: End-stage renal disease; Foxp3: Forkhead box P3; IL-10: Interleukin-10; IL-17: Interleukin-17; IL-6: Interleukin-6; LN: Lupus nephritis; SLE: Systemic lupus erythaematosus; Th17 cells: T helper 17 cells; Treg cells: Regulatory T cells; VIP: Vasoactive intestinal peptide

\section{Acknowledgements}

The authors would like to thank Springer Nature for editing the manuscript.

\section{Authors' contributions}

All authors were engaged in the experiment design. DF and SS was involved in the collection, interpretation and analysis of data, and wrote the manuscript. JW was involved in the collection and helped in the analysis of data. YY conceived the conception and design of the study, decided the direction of discussion, and helped to revise the manuscript. All authors read and approved the final manuscript.

\section{Funding}

This work was supported by the National Natural Science Foundation of China (Nos. 81560271 and 81860296), the Key Project of Scientific Research of Guangxi Colleges and Universities (No. KY2015ZD092), and the Program of the Natural Science Foundation of Guangxi (Nos. 2017GXNSFDA198005 and 2018GXNSFAA281038). The funders had no role in the design of the study and collection, analysis, and interpretation of data, nor in writing the manuscript.

\section{Availability of data and materials}

The datasets used and/or analysed during the current study are available from the corresponding author on reasonable request.

\section{Ethics approval and consent to participate}

All animal experiments that included housing and using the mice were reviewed and approved by the Animal Experiments Committee of Youjiang Medical University for Nationalities (No. 2017060501), and were performed in accordance with the guidelines for the care and use of laboratory animals established by the Committee.

Consent for publication

Not applicable.

\section{Competing interests}

The authors declare that they have no competing interests.

Received: 14 April 2019 Accepted: 29 August 2019

Published online: 05 September 2019

\section{References}

1. Maroz N, Segal MS. Lupus nephritis and end-stage kidney disease. Am J Med Sci. 2013;346(4):319-23.

2. Xing Q, Wang B, Su H, Cui J, Li J. Elevated Th17 cells are accompanied by FoxP3+ Treg cells decrease in patients with lupus nephritis. Rheumatol Int. 2012:32(4):949-58.

3. Zhu M, Mo H, Li D, Luo X, Zhang L. Th17/Treg imbalance induced by increased incidence of atherosclerosis in patients with systemic lupus erythematosus (SLE). Clin Rheumatol. 2013;32:1045-52.

4. Katsuyama T, Tsokos GC, Moulton VR. Aberrant T cell signaling and subsets in systemic lupus erythematosus. Front Immunol. 2018;9:1088.

5. Freitas EC, de Oliveira MS, Monticielo OA. Pristane-induced lupus: considerations on this experimental model. Clin Rheumatol. 2017;36(11):2403-14.

6. Chae BS, Shin TY. Immunoregulatory abnormalities of T cells and hyperreactivity of B cells in the in vitro immune response in pristaneinduced lupus mice. Arch Pharm Res. 2007;30(2):191-8.

7. Jayawardena D, Guzman G, Gill RK, Alrefai WA, Onyuksel H, Dudeja PK Expression and localization of VPAC1, the major receptor of vasoactive intestinal peptide along the length of the intestine. Am J Physiol Gastrointest Liver Physiol. 2017:313(1):G16-25.

8. Kasacka I, Piotrowska $\dot{Z}$, Janiuk I. Influence of renovascular hypertension on the distribution of vasoactive intestinal peptide in the stomach and heart of rats. Exp Biol Med. 2015;240(11):1402-7.

9. Deng $G$, Jin $L$. The effects of vasoactive intestinal peptide in neurodegenerative disorders. Neurol Res. 2017;39(1):65-72.

10. Benitez R, Delgado-Maroto V, Caro M, Forte-Lago I, Duran-Prado M, O'Valle F, Lichtman AH, Gonzalez-Rey E, Delgado M. Vasoactive intestinal peptide ameliorates acute myocarditis and atherosclerosis by regulating inflammatory and autoimmune responses. J Immunol. 2018;200(11):3697-710.

11. Villanueva-Romero R, Gutiérrez-Cañas I, Carrión M, Pérez-García S, Seoane IV, Martínez C, Gomariz RP, Juarranz Y. The anti-inflammatory mediator, vasoactive intestinal peptide, modulates the differentiation and function of th subsets in rheumatoid arthritis. J Immunol Res. 2018:2018(undefined):6043710.

12. Li C, Zhu F, Wu B, Wang Y. Vasoactive intestinal peptide protects salivary glands against structural injury and secretory dysfunction via IL-17A and AQP5 regulation in a model of Sjögren syndrome. Neuroimmunomodulation. 2018;24(6):300-9.

13. Pozo D, Anderson P, Gonzalez-Rey E. Induction of alloantigen-specific human T regulatory cells by vasoactive intestinal peptide. J Immunol. 2009; 183(7):4346-59.

14. Han S, Zhuang H, Yuan X, Lee P, Yi L, Wilson JC, Vidal O, Hong SC, Yu S, Yang LJ. Maintenance of autoantibody production in pristane-induced murine lupus. Arthritis Res Ther. 2015;17(1):1-13.

15. You $Y$, Qin $Y$, Lin $X$, Yang F, Li J, Sooranna SR, Pinhu L. Methylprednisolone attenuates lipopolysaccharide-induced fractalkine expression in kidney of lupus-prone MRL/lpr mice through the NFkappaB pathway. BMC Nephrol. 2015;16:148. 
16. Bluestone JA, Liu W, Yabu JM, Laszik ZG, Putnam A, Belingheri M, Gross DM, Townsend RM, Vincenti F. The effect of costimulatory and interleukin 2 receptor blockade on regulatory T cells in renal transplantation. Am J Transplant Off J Am Soc Transplant Am Soc Transplant Surg. 2008;8(10):2086-96.

17. Satoh M, Reeves WH. Induction of lupus-associated autoantibodies in BALB/C mice by intraperitoneal injection of pristane. J Exp Med. 1994;180(6):2341-6.

18. Arbuckle MR, McClain MT, Rubertone MV, Scofield RH, Dennis GJ, James JA, Harley JB. Development of autoantibodies before the clinical onset of systemic lupus erythematosus. N Engl J Med. 2003;349(16):1526-33.

19. Crispín JC, Liossis SN, Kistoth K, Lieberman LA, Kyttaris VC, Juang YT, Tsokos GC. Pathogenesis of human systemic lupus erythematosus: recent advances. Trends Mol Med. 2010;16(2):47-57.

20. Yang J, Chu Y, Yang X, Gao D, Zhu L, Yang X, Wan L, Li M. Th17 and natural Treg cell population dynamics in systemic lupus erythematosus. Arthritis Rheum. 2009;60(5):1472-83.

21. Delgado M, Ganea D. Vasoactive intestinal peptide: a neuropeptide with pleiotropic immune functions. Amino Acids. 2013;45(1):25-39.

22. Forghani $P$, Petersen $C T$, Waller EK. Activation of VIP signaling enhances immunosuppressive effect of MDSCs on CMV-induced adaptive immunity. Oncotarget. 2017;8(47):81873-9

23. Shi H, Carion TW, Jiang Y, Steinle JJ, Berger EA. VIP protects human retinal microvascular endothelial cells against high glucose-induced increases in TNF-a and enhances RvD1. Prostaglandins Other Lipid Mediat. 2016; 123(undefined):28-32.

24. Gomariz RP, Gutiérrez-Cañas I, Martínez C, Juarranz Y, Leceta J. New insights into the role of VIP on the ratio of T-cell subsets during the development of autoimmune diabetes. Immunol Cell Biol. 2010;88(7):734-45.

25. Tan YV, Abad C, Wang Y, Lopez R, Waschek J. VPAC2 (vasoactive intestinal peptide receptor type 2) receptor deficient mice develop exacerbated experimental autoimmune encephalomyelitis with increased Th1/Th17 and reduced Th2/Treg responses. Brain Behav Immun. 2015;44(undefined):167-75.

26. Ibrahim H, Barrow P, Foster N. VIP as a potential therapeutic agent in gram negative sepsis. Endocr Metab Immune Disord Drug Targets. 2012;12(4):308-15

27. Jayawardena D, Anbazhagan AN, Guzman G, Dudeja PK, Onyuksel H. Vasoactive intestinal peptide nanomedicine for the management of inflammatory bowel disease. Mol Pharm. 2017;14(11):3698-708.

28. Gonzalez-Rey E, Fernandez-Martin A, Chorny A, Delgado M. Vasoactive intestinal peptide induces $C D 4+, C D 25+T$ regulatory cells with therapeutic effect in collagen-induced arthritis. Arthritis Rheum. 2006; 54(3):864-76.

29. Deng S, Xi Y, Wang H, Hao J, Niu X, Li W, Tao Y, Chen G. Regulatory effect of vasoactive intestinal peptide on the balance of Treg and Th17 in collagen-induced arthritis. Cell Immunol. 2010;265(2):105-10.

\section{Publisher's Note}

Springer Nature remains neutral with regard to jurisdictional claims in published maps and institutional affiliations.

Ready to submit your research? Choose BMC and benefit from:

- fast, convenient online submission

- thorough peer review by experienced researchers in your field

- rapid publication on acceptance

- support for research data, including large and complex data types

- gold Open Access which fosters wider collaboration and increased citations

- maximum visibility for your research: over $100 \mathrm{M}$ website views per year

At $\mathrm{BMC}$, research is always in progress.

Learn more biomedcentral.com/submissions 